\title{
6 Die Hochschulen Wiens als Arbeitgeber
}

\subsection{Die Beschäftigten der Hochschulen im Überblick}

An den Wiener Hochschulen waren im Wintersemester 2011 gemäß der im Rahmen dieser Studie durchgeführten Erhebung rund 31.400 Unselbstständige (nach Kopfzahl) beschäftigt. Die Bedeutung für die Stadtökonomie ist beträchtlich: So umfassen jene beiden Sektoren - öffentliche Verwaltung sowie Erziehung und Unterricht ${ }^{1}$-, denen die Hochschulen zugerechnet werden, 157.006 Beschäftigte, wobei die Universitätsangestellten immerhin 19,9 \% ausmachen, also ein Fünftel der unselbstständig Beschäftigten in Wien. Auf die Gesamtzahl in Wien bezogen (777.173), entfallen auf die Hochschulen 4,0\% (Statistik Austria).

Die Position der größten Universitäten der Stadt im Ranking der Wiener Unternehmen nach der Beschäftigtenzahl unterstreicht deren Bedeutung als Arbeitgeber: So ist die Universität Wien mit 9.496 Beschäftigten (Kopfzahl) im Studienjahr 2011 mit Abstand größer als das größte privatwirtschaftliche Unternehmen (Uni Credit/Bank Austria mit 7.235 Beschäftigte). Bei den Einrichtungen des öffentlichen Sektors ${ }^{2}$ liegt sie nach dem Wiener Stadtschulrat (11.294 Beschäftigte) an dritter Stelle (vgl. Tabelle 6.1 und 6.2). Aber auch die Medizinische Universität (2011: 5.372 Beschäftigte) liegt in Wien immerhin vor dem drittgrößten privatwirtschaftlichen Unternehmen (Billa AG mit 4.479 Beschäftigte), die TU Wien mit 4.536 Beschäftigten ebenso. Sowohl nach der Arbeitskräftestatistik als auch nach dem Kriterium der Unternehmensgröße sind die Wiener Hochschulen ein wichtiger Arbeitgeber und damit auch Wirtschaftsfaktor für die städtische Ökonomie, wobei für die Wertschöpfungseffekte die innere Struktur der Einrichtungen zu berücksichtigen ist.

Das Wertschöpfungspotential der Wiener Hochschulen lässt sich nicht nur auf Basis der Beschäftigtenzahl messen, sondern noch vielmehr anhand des naturgemäß hohen Qualifizierungsniveaus bzw. der Akademikerquote: Rund zwei Drittel der Beschäftigten $(65,9 \%)$ zählen als wissenschaftliches Personal, doch auch beim allgemeinen Personal beträgt die Akademikerquote durchschnittlich 33,5 \% (vgl. Tabelle 6.3). Zwischen den drei Hochschultypen schwankt die Beschäftigtenstruktur erheblich. So ist der Anteil des allgemeinen Personals sehr unterschiedlich und liegt bei den Fachhochschulen mit $41,0 \%$ deutlich über dem Durchschnitt. Doch auch variierende Akademikerquoten beim allgemeinen Personal zeigen, dass die Hochschultypen über sehr unterschiedliche Organisationsstrukturen verfügen.

1 Nace-08-Sektoren O (öffentl. Verwaltung, Verteidigung, Sozialversicherung) und P (Erziehung und Unterricht).

2 Bei Einrichtungen des öffentlichen Sektors ist die direkte Vergleichbarkeit aus mehreren Gründen eingeschränkt: Erstens sind die Arbeitnehmer bei unterschiedlichen Sozialversicherungsträgern versichert (neben der BVA und der Wiener Gebietskrankenkasse etwa die Krankenfürsorgeanstalt der Bediensteten der Stadt Wien). Zweitens wurden nur jene Einrichtungen angeführt, deren Beschäftigte dem Standort Wien zugerechnet werden können. 
Tabelle 6.1: Größte Unternehmen der Stadt Wien nach der Beschäftigtenzahl 2011

\begin{tabular}{clc}
\hline Rang & Unternehmen & Beschäftigte* \\
\hline 1 & Unicredit Bank Austria AG & 7.235 \\
2 & Siemens AG & 6.045 \\
3 & Billa AG & 4.479 \\
4 & Österreichische Post & 3.354 \\
5 & Telekom Austria & 3.215 \\
6 & Merkur Warenhandels-GmbH & 2.882 \\
7 & Spar Österreichische Warenhandelsgesellschaft & 2.823 \\
8 & Erste Bank & 2.736 \\
9 & Bawag & 2.551 \\
10 & Baxter AG & 2.404 \\
\hline
\end{tabular}

*) Beschäftigtenzahl (keine Vollzeitäquivalente, jedoch ohne geringfügig Beschäftigte)

Quelle: Statistik Wiener Gebietskrankenkasse.

Tabelle 6.2: Größte Einrichtungen des öffentlichen Sektors der Stadt Wien nach der Beschäftigtenzahl 2011

\begin{tabular}{clc}
\hline Rang Unternehmen & Beschäftigte* $^{*}$ \\
\hline 1 & Stadt Wien & 21.358 \\
2 & Stadtschulrat für Wien & 11.294 \\
3 & Bundespolizeidirektion Wien & 7.618 \\
4 & BM für Landesverteidigung und Sport & 4.931 \\
5 & Wiener Gebietskrankenkasse & 3.758 \\
6 & BM für Unterricht, Kunst und Kultur & 3.506 \\
7 & Wiener Wohnen & 2.990 \\
8 & BM für Inneres & 2.746 \\
9 & BM für Land- und Forstwirtschaft & 2.415 \\
10 & Österreichischer Rundfunk & 2.292 \\
\hline
\end{tabular}

*) Beschäftigtenzahl (keine Vollzeitäquivalente, jedoch ohne geringfügig Beschäftigte)

Quelle: Statistik Wiener Gebietskrankenkasse; BVA.

Auch beim wissenschaftlichen Personal, das hier grob in Professoren/Habilitierte, Postdocs (inklusive Tenure-Track, also Laufbahnstellen) sowie befristete Doktorandenstellen und externe Lehrende untergliedert ist, zeigen sich deutliche Unterschiede. Die letztgenannte Gruppe fällt nicht zuletzt deshalb stark ins Gewicht, weil es sich hier überwiegend um Teilzeitstellen handelt und diese Statistik auf der Kopfzahl und nicht 
auf Vollzeitäquivalenten basiert. Auch hier drückt sich die für Hochschulen typische, hierarchische Heterogenität der Beschäftigungsverhältnisse, aber auch die Strukturunterschiede - etwa der Anteil der Professoren/Habilitierten am gesamten wissenschaftlichen Personal - aus.

Tabelle 6.3: Differenzierung der Beschäftigten an den Wiener Hochschulen 2011

\begin{tabular}{|c|c|c|c|c|c|}
\hline & \multicolumn{5}{|c|}{ Allgemeines Personal } \\
\hline & Akademiker & Nichtakademiker & Gesamt & $\begin{array}{c}\text { Akademiker- } \\
\text { quote }(\%)\end{array}$ & $\begin{array}{l}\text { Anteil allgemeines } \\
\text { Personal (\%) }\end{array}$ \\
\hline Universitäten & 2.818 & 6.204 & 9.022 & 31,2 & 33,3 \\
\hline Fachhochschulen & 695 & 849 & 1.544 & 45,0 & 41,0 \\
\hline Privatuniversitäten & 78 & 87 & 166 & 47,2 & 30,1 \\
\hline \multirow[t]{3}{*}{ Gesamt } & 3.592 & 7.140 & 10.732 & 33,5 & 34,1 \\
\hline & \multicolumn{5}{|c|}{ Wissenschaftliches Personal } \\
\hline & Prof./Hab. & Postdocs & Sonstige* & Gesamt & $\begin{array}{c}\text { Anteil Prof./Hab. } \\
(\%)\end{array}$ \\
\hline Universitäten & 2.632 & 1.758 & 13.721 & 18.111 & 14,5 \\
\hline Fachhochschulen & 280 & 254 & 1.687 & 2.222 & 12,6 \\
\hline Privatuniversitäten & 106 & 39 & 241 & 385 & 27,5 \\
\hline Gesamt & 3.018 & 2.051 & 15.649 & 20.718 & 14,6 \\
\hline
\end{tabular}

Quellen: eigene Erhebung 2013; eigene Berechnungen.

*) Sonstige: Prädocs, externe Lehrkräfte o.Ä.

\subsection{Der Wohnort der Beschäftigten}

Wo leben die Beschäftigten der Wiener Hochschulen? Die Frage des Wohnortes ist insofern relevant, als dieser das Ausgabeverhalten und damit auch die Wertschöpfungseffekte für die Stadt Wien beeinflusst. Auf Grundlage der in dieser Studie durchgeführten Abgrenzung des Wiener Umlandes wurden die Beschäftigten drei Raumeinheiten zugeteilt: der Kernstadt Wien, dem Wiener Umland sowie dem restlichen Österreich, wobei auch Beschäftigte aus dem nahen Ausland (insbesondere der Westslowakei) einpendeln. Wie bei den Studierenden wurde auch hier angenommen (vgl. Kapitel 5.2), dass die im Wiener Umland wohnhaften Beschäftigten täglich an den Arbeitsort nach Wien pendeln, während jene Beschäftigten, deren Wohnort außerhalb des Stadtumlandes liegt, wochenweise pendeln und die meiste Zeit in Wien verbringen. Die letztgenannte Gruppe wird wie auch bei den Studierenden hinsichtlich der Wertschöpfungseffekte mit den in Wien lebenden Beschäftigten gleichgesetzt. 
Gemäß dieser regionalen Zuordnung der Beschäftigten zeigt sich, dass beim wissenschaftlichen Personal eine Suburbanisierungsrate von $10,7 \%$ vorliegt, wobei die Gruppe der Professoren/Habilitierten einen leicht höheren Wert aufweist (vgl. Tabelle 6.4). Beim allgemeinen Personal ist der Anteil der im Wiener Umland Lebenden beinahe doppelt so hoch (19,3\%), wobei vor allem die Nichtakademiker $(22,4 \%)$ für den deutlichen Unterschied ausschlaggebend sind. Während also beim wissenschaftlichen Personal die Suburbanisierungsrate mit dem Qualifikationsniveau steigt, ist dieser Zusammenhang beim allgemeinen Personal genau umgekehrt. Eine Differenzierung nach den Hochschultypen hat nur minimale Abweichungen von dem hier beschriebenen Muster ergeben, da der persönliche Lebensstil und die Einkommenssituation wohl die ausschlaggebenden Erklärungsfaktoren darstellen.

Tabelle 6.4: Die Wohnorte der Beschäftigten an den Wiener Hochschulen 2011

\begin{tabular}{lrcccc}
\hline & Wien & Wiener Umland & Sonstige & Gesamt & $\begin{array}{c}\text { Anteil } \\
\text { Wiener Umland (\%) }\end{array}$ \\
\hline Wiss. Personal & 16.627 & 2.217 & 1.875 & 20.718 & $10,7 \%$ \\
Hab./Prof. & 3.168 & 497 & 457 & 4.122 & $12,1 \%$ \\
Postdocs & 1.675 & 215 & 158 & 2.048 & $10,5 \%$ \\
Rest & 11.784 & 1.505 & 1.260 & 14.548 & $10,3 \%$ \\
\hline Allgemeines Personal & 7.662 & 2.071 & 999 & 10.732 & $19,3 \%$ \\
Akademiker & 2.879 & 472 & 245 & 3.596 & $13,1 \%$ \\
Nichtakademiker & 4.783 & 1.599 & 754 & 7.136 & $22,4 \%$ \\
\hline
\end{tabular}

Quellen: eigene Erhebung 2013; uni:data 2013; eigene Berechnungen.

\subsection{Die Staatsbürgerschaften der Beschäftigten}

Die Wiener Hochschulen sind nicht nur als tertiäre Ausbildungsstätte, sondern auch als Arbeitgeber ein wichtiger Faktor für die Zuwanderung hochqualifizierter ausländischer Arbeitskräfte und beeinflussen somit das Migrationsregime der Stadt in positiver Weise. Der mittlerweile seit mehreren Jahren andauernde Trend zur Internationalisierung der Hochschulen betrifft nicht nur die Studierenden, sondern ebenso die dort Beschäftigten. In der Folge werden die Entwicklung sowie die soziale und räumliche Struktur der allgemeinen und wissenschaftlichen Mitarbeiter an den drei Hochschultypen differenziert vorgestellt.

\section{Die Internationalisierung des allgemeinen Hochschulpersonals}

Der Anteil der ausländischen Staatsbürger beim allgemeinen Hochschulpersonal liegt bei 10,3 \%, allerdings existieren zwischen den Hochschultypen sowie dem Qualifikationsniveau beträchtliche Unterschiede. So besteht bei den Universitäten ein deutlicher 
Abbildung 6.1: Staatszugehörigkeit des allgemeinen Personals 2009-2011 an den Universitäten Wiens

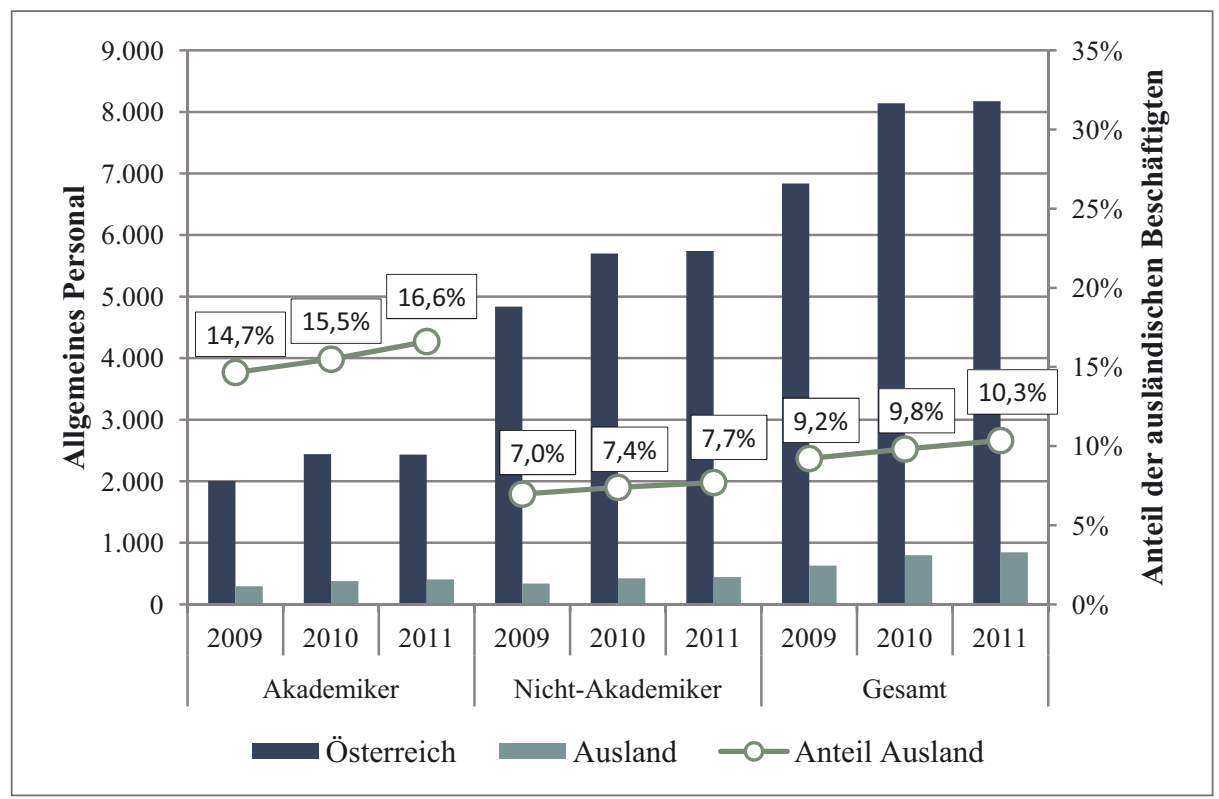

Quellen: eigene Erhebung 2013; uni:data 2013; eigene Berechnungen.

Unterschied zwischen akademischen und nichtakademischen Beschäftigten (vgl. Abbildung 6.1): Bei den Akademikern ist der Anteil ausländischer Beschäftigter mit 16,6 \% nicht nur doppelt so hoch wie bei den Nichtakademikern (7,7 \%), in den Jahren 2009 bis 2011 ist deren Anteil auch überproportional stark gestiegen, wenngleich in Summe die Wachstumsdynamik weit hinter jener der ausländischen Studierenden liegt. Es zeigt sich damit, dass die Universitäten vor allem im Bereich der hochqualifizierten Arbeitnehmer ausländische Arbeitskräfte anziehen.

Im Segment der Fachhochschulen liegt der Anteil der ausländischen Beschäftigten im nichtwissenschaftlichen Bereich (2011: 5,1 \%) deutlich unter jenem der Universitäten (2011: 10,3\%), wobei es seit 2009 zu einem (leichten) Rückgang des Anteils sowie auch der absoluten Beschäftigtenzahl gekommen ist (vgl. Abbildung 6.2). Wie auch bei den Universitäten zeigt sich bei den Akademikern eine höhere, wenngleich sinkende Quote der ausländischen Beschäftigten. In der Kategorie der Privatuniversitäten (vgl. Abbildung 6.3) lässt sich hingegen ein wesentlich stärkeres Internationalisierungsniveau des allgemeinen Personals feststellen - rund jeder Dritte $(28,5 \%)$ ist ausländischer Staatsbürger. Hier zeigt sich überdies insofern ein gegenläufiger Trend, als dass der hohe Anteil der ausländischen Staatsbürger beim akademischen Personal seit 2009 massiv gesunken ist und umgekehrt bei den Nichtakademikern bis 2011 auf 33,2 \% 
Abbildung 6.2: Staatszugehörigkeit des allgemeinen Personals 2009-2011 an den Fachhochschulen Wiens

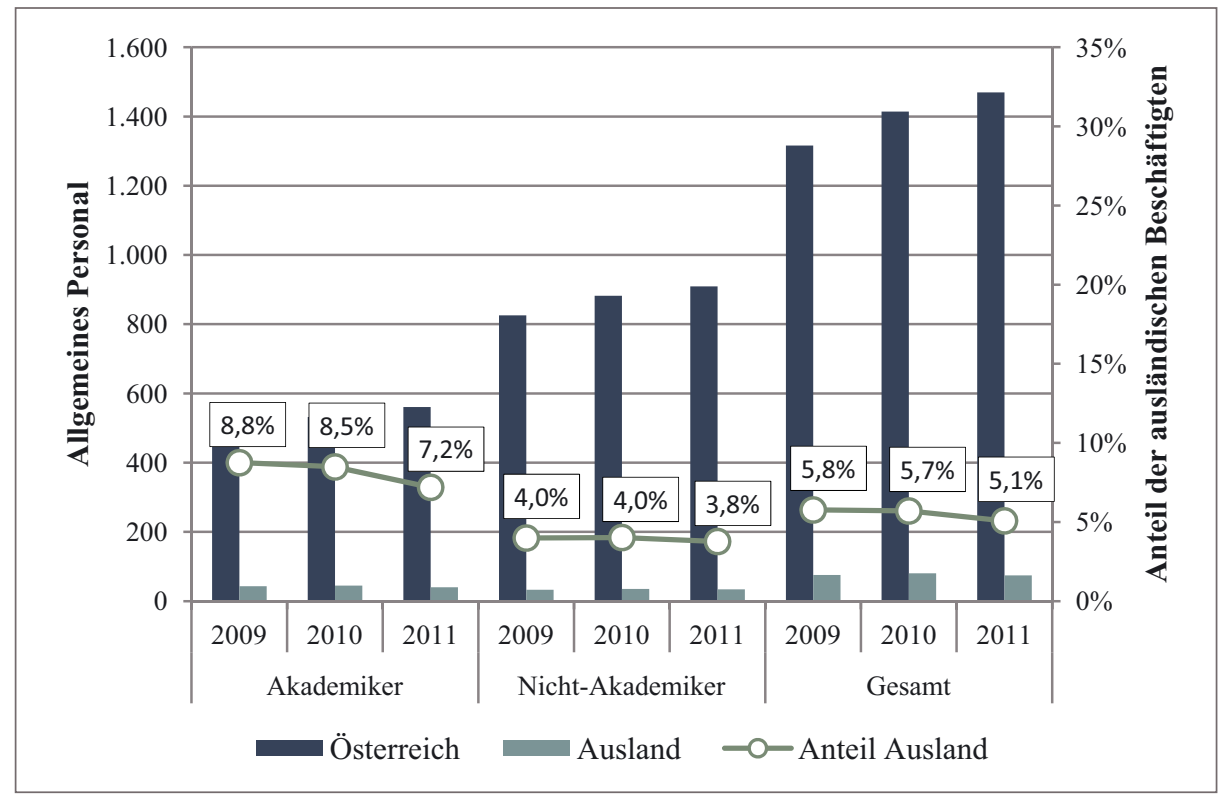

Quellen: eigene Erhebung 2013; uni:data 2013; eigene Berechnungen.

gestiegen ist. Aufgrund der geringen Fallzahl (53 ausländische Staatsbürger im allgemeinen Personal) ist diese Entwicklung für den gesamten Hochschulsektor allerdings wenig relevant und kann sich überdies auch rasch wieder ändern.

\section{Die Internationalisierung des wissenschaftlichen Hochschulpersonals}

In der Gruppe des wissenschaftlichen Personals an den Hochschulen lässt sich eine wesentlich stärkere Internationalisierung als beim allgemeinen Personal feststellen. Das hohe quantitative Niveau - an den Universitäten hat knapp jeder vierte wissenschaftliche Angestellte eine ausländische Staatsbürgerschaft - sowie das starke Wachstum sind Ausdruck einer zunehmenden Internationalisierung der Wissenschaftslandschaft, die - im internationalen Maßstab vielleicht etwas verzögert - die österreichische und Wiener Hochschullandschaft erreicht hat.

An den Universitäten bestätigt sich der deutliche Trend zur Internationalisierung insofern, als der Beschäftigtenzuwachs ausschließlich durch die steigende Zahl ausländischer Staatsbürger erzielt wird (vgl. Tabelle 6.5): Die Zahl der inländischen Beschäftigten stagnierte, während jene der ausländischen Beschäftigten um 786 angestiegen ist, womit sich der Anteil der ausländischen Staatsbürger von 21,4 \% auf 24,8 \% verschob. Dieser Trend lässt sich auf allen drei Qualifizierungsstufen feststellen, wobei sich doch 
Abbildung 6.3: Staatszugehörigkeit des allgemeinen Personals 2009-2011 an den Privatuniversitäten Wiens

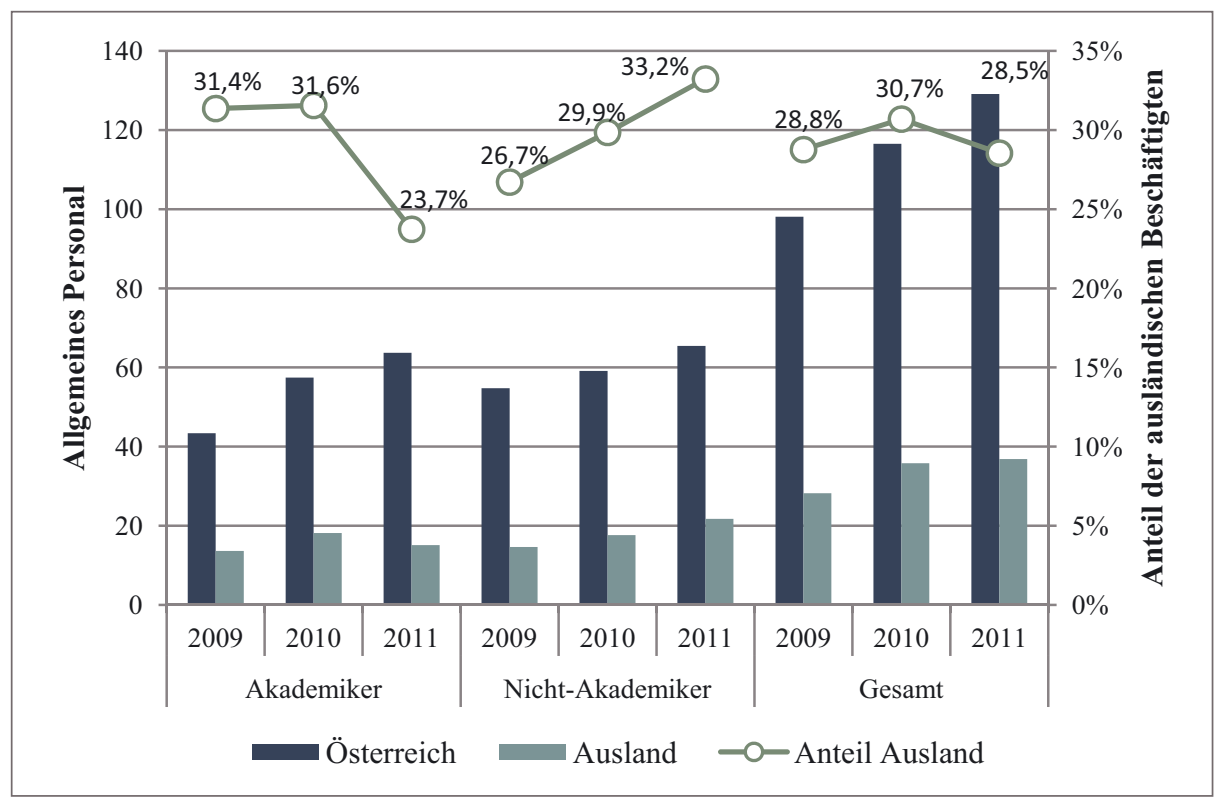

Quellen: eigene Erhebung 2013; uni:data 2013; eigene Berechnungen.

gewisse Unterschiede zeigen: Bei Prae-Docs und sonstigem externem Lehrpersonal ist der Anteil mit 23,5\% leicht unterdurchschnittlich, bei Professoren/Habilitierten (27,7\%), insbesondere aber bei den Postdocs (30,7 \%), liegt er über dem Durchschnittswert. Hier ist allerdings festzuhalten, dass diese Internationalisierung bei den Professoren/ Habilitierten die Folge eines Generationenwechsels ist, da nach altem Universitätsrecht aus dem Ausland berufene Professoren ,nostrifiziert“" wurden, also die österreichische Staatsbürgerschaft erhielten. Tendenziell nimmt mit steigendem Qualifizierungsniveau der Internationalisierungsgrad zu, insbesondere wenn man auch das allgemeine Personal berücksichtigt. Vergleicht man diese Entwicklung mit der Internationalisierung bei den Studierenden (vgl. Kapitel 5.3), so zeigt sich, dass diese beim wissenschaftlichen Universitätspersonal insgesamt auf ähnlichem Niveau liegt.

An den Fachhochschulen ist das Internationalisierungsniveau - auch bei den Studierenden sowie beim allgemeinen Personal - deutlich niedriger als an den Universitäten. Die stärkere Orientierung der Ausbildung am Praxisbezug sowie an den Erfordernissen des heimischen Arbeitsmarktes dürfte hier eine gewisse Rolle spielen. Im Jahr 2011 hatten nur 5,2 \% des wissenschaftlichen Personals eine ausländische Staatsbürgerschaft, absolut stieg deren Zahl seit 2009 gerade einmal um 47 Beschäftigte. Zwischen den drei Qualifizierungsstufen bestehen jedoch beträchtliche Unterschiede: Während bei Professoren/Habilitierten der Anteil ausländischer Staatsbürger bei nur 2,9 \% liegt 
Tabelle 6.5: Staatsbürgerschaft des wissenschaftlichen Personals an den Wiener Hochschulen 2009 bis 2011

\begin{tabular}{|c|c|c|c|c|c|}
\hline \multicolumn{6}{|c|}{ Universitäten } \\
\hline & & Prof./Hab. & Postdocs & Rest & Gesamt \\
\hline \multirow{3}{*}{ ஓे } & Österreich & 2.059 & 1.175 & 10.376 & 13.610 \\
\hline & Ausland & 647 & 371 & 2.687 & 3.705 \\
\hline & Anteil Ausland (\%) & 23,9 & 24,0 & 20,6 & 21,4 \\
\hline \multirow{3}{*}{$\frac{\circ}{\stackrel{\circ}{\sim}}$} & Österreich & 1.919 & 1.136 & 10.530 & 13.584 \\
\hline & Ausland & 700 & 456 & 2.959 & 4.115 \\
\hline & Anteil Ausland (\%) & 26,7 & 28,6 & 21,9 & 23,2 \\
\hline \multirow{3}{*}{$\overline{\bar{\delta}}$} & Österreich & 1.903 & 1.219 & 1.0497 & 13.619 \\
\hline & Ausland & 729 & 539 & 3224 & 4.492 \\
\hline & Anteil Ausland (\%) & 27,7 & 30,7 & 23,5 & 24,8 \\
\hline
\end{tabular}

\begin{tabular}{|c|c|c|c|c|c|}
\hline \multicolumn{6}{|c|}{ Fachhochschulen } \\
\hline & & Prof./Hab. & Postdocs & Rest & Gesamt \\
\hline \multirow{3}{*}{ ஓे } & Österreich & 323 & 245 & 1.126 & 1.694 \\
\hline & Ausland & 0 & 53 & 15 & 68 \\
\hline & Anteil Ausland (\%) & 0,0 & 17,7 & 1,3 & 3,9 \\
\hline \multirow{3}{*}{$\frac{\circ}{\stackrel{\circ}{\circ}}$} & Österreich & 374 & 254 & 1.294 & 1.921 \\
\hline & Ausland & 12 & 55 & 24 & 91 \\
\hline & Anteil Ausland (\%) & 3,2 & 17,9 & 1,8 & 4,5 \\
\hline \multirow{3}{*}{$\underset{\sim}{\bar{\nu}}$} & Österreich & 272 & 196 & 1.639 & 2.107 \\
\hline & Ausland & 8 & 58 & 48 & 115 \\
\hline & Anteil Ausland (\%) & 2,9 & 22,8 & 2,8 & 5,2 \\
\hline
\end{tabular}

\begin{tabular}{|c|c|c|c|c|c|}
\hline \multicolumn{6}{|c|}{ Privatuniversitäten } \\
\hline & & Prof./Hab. & Postdocs & Rest & Gesamt \\
\hline \multirow{3}{*}{ ஓे } & Österreich & 48 & 15 & 203 & 266 \\
\hline & Ausland & 37 & 9 & 48 & 94 \\
\hline & Anteil Ausland (\%) & 43,8 & 37,0 & 19,1 & 26,2 \\
\hline \multirow{3}{*}{$\frac{\circ}{\stackrel{0}{\sim}}$} & Österreich & 58 & 21 & 191 & 270 \\
\hline & Ausland & 46 & 12 & 54 & 112 \\
\hline & Anteil Ausland (\%) & 44,4 & 35,1 & 22,2 & 29,4 \\
\hline \multirow{3}{*}{$\overline{\widetilde{O}}$} & Österreich & 56 & 27 & 189 & 271 \\
\hline & Ausland & 50 & 12 & 52 & 114 \\
\hline & Anteil Ausland (\%) & 47,1 & 30,0 & 21,6 & 29,6 \\
\hline
\end{tabular}

Quellen: eigene Erhebung 2013; eigene Berechnungen. 
(8 in absoluten Zahlen), trifft dies auf mehr als jeden fünften Postdoc zu (22,8 \%). Auch beim nichtpromovierten bzw. externen Lehrpersonal ist der Anteil der Personen mit ausländischer Staatsbürgerschaft mit 2,8 \% verschwindend gering.

Deutlich anders stellt sich die Situation hingegen für die Privatuniversitäten dar. Wenngleich die geringen Beschäftigtenzahlen wenig Einfluss auf die Gesamtstruktur der Beschäftigten an den Wiener Hochschulen haben, so ist die starke internationale Orientierung doch sehr auffällig. So war 2011 nahezu jeder Dritte (29,6\%) der 385 wissenschaftlichen Mitarbeiter ausländischer Staatsbürger, wobei die Internationalisierungsquote hier ganz klar mit dem Qualifizierungsniveau ansteigt. So besitzt bei den Professoren/Habilitierten knapp die Hälfte (47,1 \%) eine ausländische Staatsbürgerschaft, wobei in dieser Gruppe der Anteil gegenüber 2009 am stärksten gestiegen ist.

Die deutlichen Unterschiede hinsichtlich des Internationalisierungsniveaus zwischen den drei Hochschultypen unterstreichen deren Verschiedenartigkeit hinsichtlich der Orientierung in Forschung und Lehre.

\section{Die geographische Herkunft des ausländischen wissenschaftlichen Hochschulpersonals}

Die Herkunft des wissenschaftlichen Personals mit nichtösterreichischen Staatsbürgerschaften weist wie auch bei den Studierenden eine beträchtliche räumliche Konzentration auf, wenngleich auch Unterschiede hinsichtlich der Herkunft nachweisbar sind. Für eine Teilgruppe von elf Hochschulen ${ }^{3}$ lassen sich dazu Aussagen machen. Diese Einrichtungen zählen 2.796 wissenschaftliche Mitarbieter mit ausländischer Staatsbürgerschaft aus weltweit 82 Ländern. Die beträchtliche Konzentration der Herkunftsländer lässt sich jedoch daran ermessen, dass auf die Top-20-Herkunftsländer 87,0 \% und auf die Top-4-Herkunftsländer immerhin noch 60,8 \% des ausländischen Personals entfallen (vgl. Tabelle 6.6). Wichtigste Gruppe dabei sind - wie auch bei den Studierenden - deutsche Staatsbürger, die beinahe die Hälfte (47,3\%) des ausländischen wissenschaftlichen Hochschulpersonals ausmachen. Auch hier zeigt sich, dass mit zunehmendem Qualifikationsniveau der Anteil deutlich ansteigt: Deutsche Staatsbürger machen mehr als zwei Drittel (67,9\%) der ausländischen Professoren/Habilitierten aus. Die 255 deutschen Professorinnen und Professoren repräsentieren 2011 16,7 \% aller Professoren/Habilitierten an den hier berücksichtigten elf Wiener Hochschulen. Hinter Deutschland folgt mit deutlichem Abstand an zweiter Stelle Italien, aus dem 7,4 \% des ausländischen Hochschulpersonals stammten, wobei - wie bei den meisten Herkunftsländern - der Anteil mit steigendem Qualifikationsniveau abnimmt.

Hinsichtlich der Verteilung der restlichen Top-20-Herkunftsländer fällt deutlich auf, dass etwa die klassischen Gastarbeiterländer - mit Ausnahme Kroatiens auf Rang 13 - gar nicht aufscheinen - ein sehr auffälliger Unterschied gegenüber den Herkunftslän-

3 Univ. Wien, Univ. f. Bodenkultur, Veterinärmedizinische Univ., Wirtschaftsuniv., Akademie der bildenden Künste, FH Campus, Porsch FernFH, FH der WK Wien, Modul Univ., Konversatorium, Sigmund Freud Privatuniversität. 
dern der ausländischen Studierenden. So liegt Serbien mit 21 Wissenschaftlern nur auf Rang 23, unmittelbar vor der Türkei mit 20 Wissenschaftlern auf Rang 24. Dafür finden sich wichtige westeuropäische Wissenschaftsnationen (Großbritannien, Frankreich, Schweiz, Niederlande) sowie osteuropäische Staaten unter den wichtigsten Herkunftsländern (vgl. Tabelle 6.6). Überraschend sind auch die USA als wichtigstes außereuro-

Tabelle 6.6: Die 20 wichtigsten Herkunftsländer des wissenschaftlichen Personals an ausgewählten Wiener Hochschulen 2011

\begin{tabular}{|c|c|c|c|c|c|c|c|c|}
\hline & \multicolumn{2}{|c|}{ Prof./Hab. } & \multicolumn{2}{|c|}{ Postdocs } & \multicolumn{2}{|c|}{ Sonstige } & \multicolumn{2}{|c|}{ Gesamt } \\
\hline & absolut & in $\%$ & absolut & in $\%$ & absolut & in $\%$ & absolut & in $\%$ \\
\hline Gesamt & 1.520 & 100,0 & 1.325 & 100,0 & 9.331 & 100,0 & 12.176 & 100,0 \\
\hline Österreich & 1.145 & 75,3 & 947 & 71,5 & 7.288 & 78,1 & 9.381 & 77,0 \\
\hline Ausland & 376 & 24,7 & 377 & 28,5 & 2.043 & 21,9 & 2.796 & 23,0 \\
\hline Deutschland & 255 & 67,9 & 182 & 48,2 & 886 & 43,4 & 1.323 & 47,3 \\
\hline Italien & 11 & 3,0 & 31 & 8,3 & 165 & 8,1 & 208 & 7,4 \\
\hline Vereinigte Staaten & 18 & 4,8 & 10 & 2,7 & 71 & 3,5 & 99 & 3,5 \\
\hline Großbritannien & 12 & 3,1 & 8 & 2,0 & 54 & 2,6 & 73 & 2,6 \\
\hline Polen & 4 & 1,1 & 5 & 1,3 & 59 & 2,9 & 69 & 2,5 \\
\hline Frankreich & 6 & 1,6 & 7 & 1,9 & 53 & 2,6 & 66 & 2,4 \\
\hline Ungarn & 6 & 1,6 & 11 & 3,0 & 45 & 2,2 & 62 & 2,2 \\
\hline Schweiz & 12 & 3,2 & 15 & 4,0 & 34 & 1,7 & 61 & 2,2 \\
\hline Slowakei & 0 & 0,0 & 11 & 2,9 & 49 & 2,4 & 61 & 2,2 \\
\hline Spanien & 5 & 1,4 & 8 & 2,1 & 42 & 2,1 & 56 & 2,0 \\
\hline Russland & 3 & 0,8 & 6 & 1,6 & 46 & 2,3 & 55 & 2,0 \\
\hline Niederlande & 8 & 2,2 & 6 & 1,6 & 28 & 1,4 & 43 & 1,5 \\
\hline Kroatien & 2 & 0,6 & 7 & 1,9 & 31 & 1,5 & 41 & 1,4 \\
\hline Tschechische Republik & 2 & 0,5 & 7 & 1,9 & 27 & 1,3 & 36 & 1,3 \\
\hline Rumänien & 2 & 0,5 & 4 & 1,1 & 30 & 1,5 & 36 & 1,3 \\
\hline China & 0 & 0,0 & 1 & 0,3 & 34 & 1,7 & 35 & 1,3 \\
\hline Bulgarien & 3 & 0,8 & 3 & 0,8 & 26 & 1,3 & 32 & 1,1 \\
\hline Indien & 0 & 0,0 & 4 & 1,1 & 26 & 1,3 & 30 & 1,1 \\
\hline Griechenland & 0 & 0,0 & 1 & 0,3 & 24 & 1,2 & 25 & 0,9 \\
\hline Japan & 4 & 1,1 & 2 & 0,5 & 18 & 0,9 & 24 & 0,9 \\
\hline Top 20 gesamt & 354 & 94,3 & 330 & 87,4 & 1.749 & 85,6 & 2.433 & 87,0 \\
\hline Sonstige & 22 & 5,7 & 47 & 12,6 & 294 & 14,4 & 363 & 13,0 \\
\hline
\end{tabular}

Quelle: eigene Erhebung 2013. 
päisches Herkunftsland auf Rang 3 (3,5\%); andere außereuropäische Staaten liegen auf den Rängen 16 (China), 18 (Indien) und 20 (Japan) und dominieren vor allem bei der Zahl der Prädocs. Die Wiener Hochschulen scheinen sich hinsichtlich ihres internationalen Einzugsgebietes bei Studierenden und wissenschaftlichem Personal - abseits der Dominanz deutscher Staatsbürger - deutlich voneinander zu unterscheiden.

Aus einer globalen Perspektive wird die Dominanz Europas als Einzugsgebiet für den internationalen Arbeitsmarkt der Wiener Hochschulen deutlich. Außerhalb Europas spielen nur 45 Staaten als Herkunftsländer für das wissenschaftliche Personal eine (wenn auch nur geringe) Rolle (vgl. Tabelle 6.7), wobei vor allem Afrika, Westasien bzw. der arabische Raum, SO-Asien und Mittelamerika von geringer Bedeutung sind. Karte 6.2 spiegelt die Hierarchie der Staaten hinsichtlich ihrer demographischen und/ oder ökonomischen Bedeutung (insbesondere die Bric-Staaten) sowie ihrer Stellung im globalen Wissenschaftssystem und damit auch die überragende Rolle des außereuropäischen, englischen Sprachraumes wider.

Tabelle 6.7: Herkunft des ausländischen wissenschaftlichen Personals 2011 nach Kontinenten/Weltregionen für alle Hochschulen Wiens

\begin{tabular}{|c|c|c|c|c|}
\hline & Anzahl Staaten & Prof./Hab. & Postdocs & Sonstige \\
\hline Europa & 37 & 710 & 535 & 2.824 \\
\hline Osteuropa & 11 & 59 & 74 & 489 \\
\hline Nordeuropa & 9 & 30 & 25 & 160 \\
\hline Südeuropa & 10 & 56 & 91 & 491 \\
\hline Westeuropa & 7 & 565 & 345 & 1.683 \\
\hline Afrika & 12 & 0 & 6 & 19 \\
\hline Nord- und Westafrika & 6 & 0 & 4 & 10 \\
\hline Zentral-, Ost- und Südafrika & 6 & 0 & 2 & 9 \\
\hline Amerika & 12 & 47 & 25 & 216 \\
\hline Nordamerika & 2 & 45 & 17 & 154 \\
\hline Mittelamerika und Karibik & 3 & 1 & 2 & 19 \\
\hline Südamerika & 7 & 1 & 5 & 43 \\
\hline Asien & 19 & 25 & 38 & 248 \\
\hline Zentralasien & 2 & 0 & 1 & 3 \\
\hline Westasien & 3 & 9 & 6 & 49 \\
\hline Süd-, Ost- und Südostasien & 14 & 16 & 31 & 196 \\
\hline Ozeanien & 2 & 5 & 5 & 17 \\
\hline Gesamt & 82 & 787 & 609 & 3.324 \\
\hline
\end{tabular}

Quellen: eigene Erhebung 2013; uni:data 2013; eigene Berechnungen. 
Karte 6.1: Herkunft des wissenschaftlichen Personals an Wiener Hochschulen 2011 innerhalb Europas

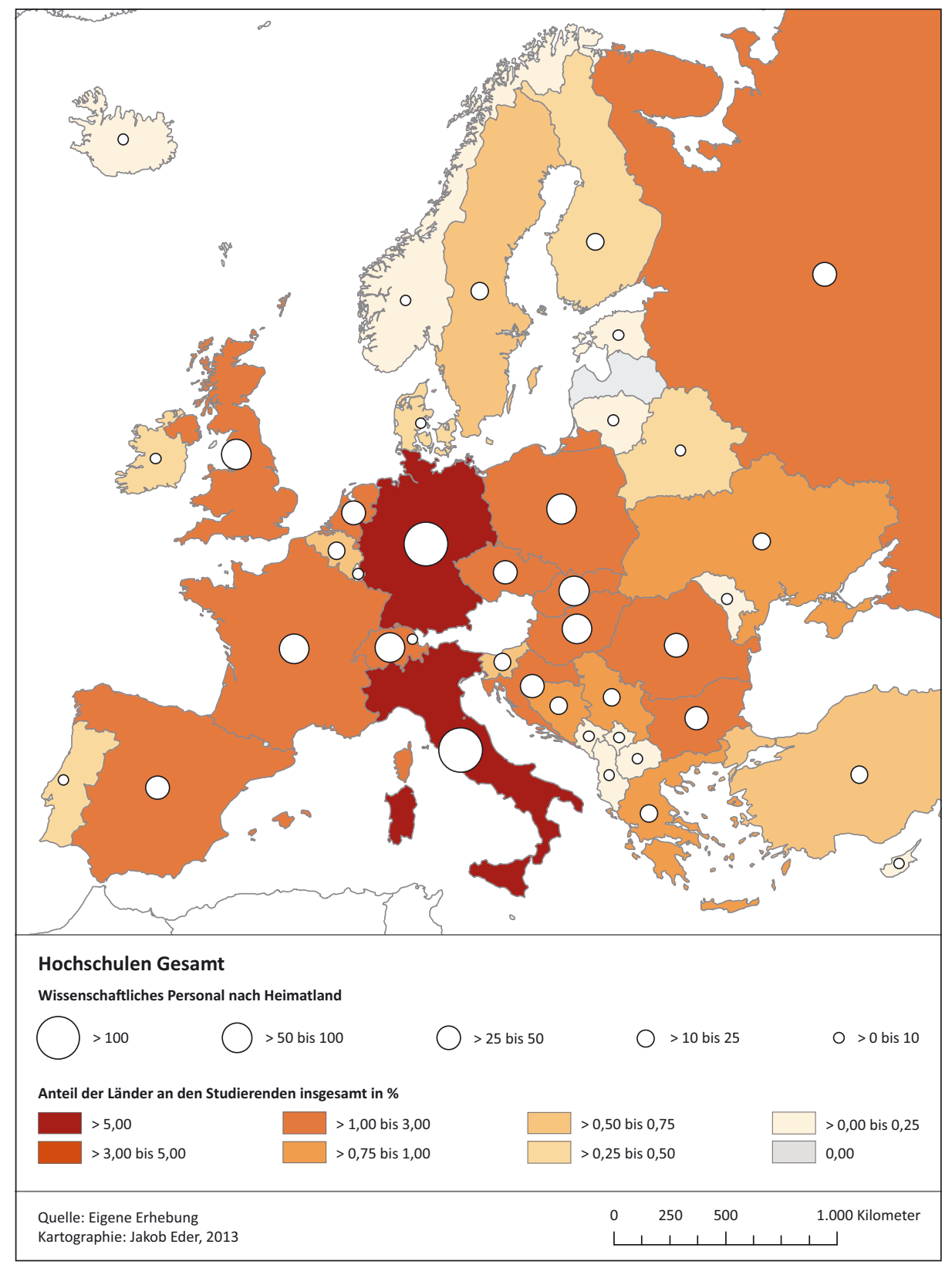


Karte 6.2: Weltweite Herkunft des wissenschaftlichen Personals an Wiener Hochschulen 2011

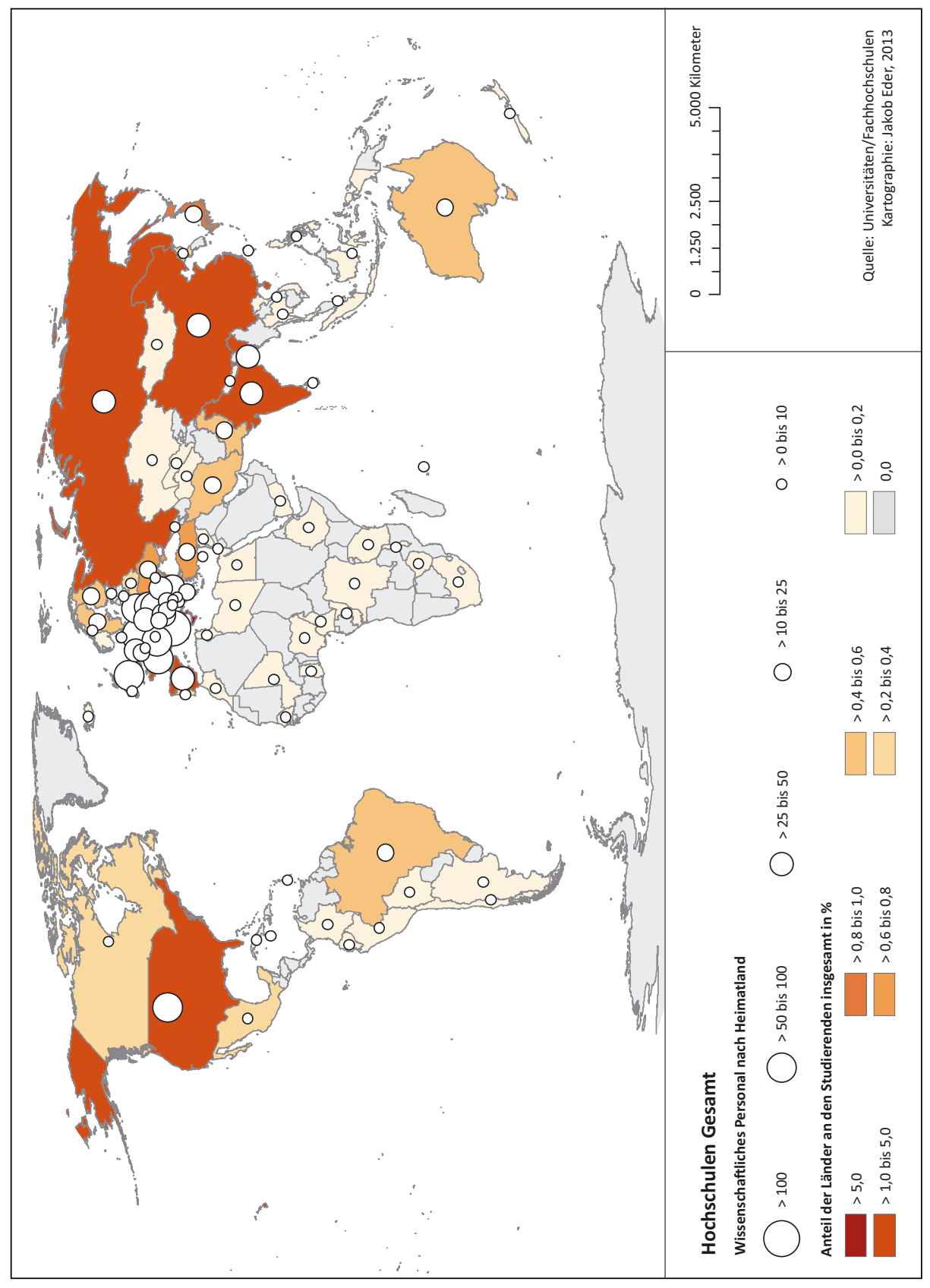

